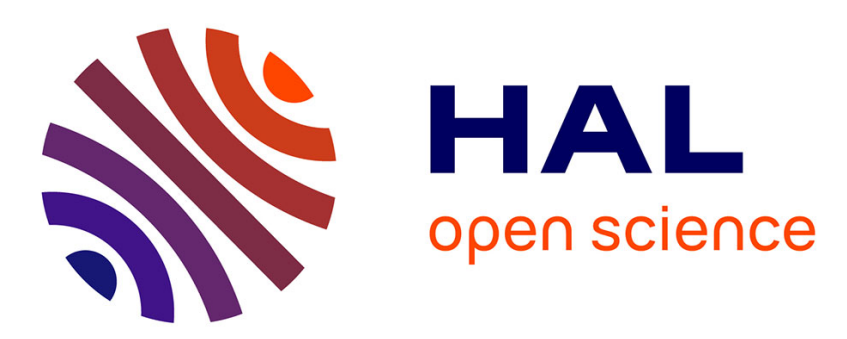

\title{
Treating Moving Interfaces in Thermal Models with the C-NEM
}

Julien Yvonnet, David Ryckelynck, Philippe Lorong, Francisco Chinesta

\section{To cite this version:}

Julien Yvonnet, David Ryckelynck, Philippe Lorong, Francisco Chinesta. Treating Moving Interfaces in Thermal Models with the C-NEM. Meshfree Methods for Partial Differential Equations II. Lecture Notes in Computational Science and Engineering, 43, pp.255-269, 2005, 10.1007/3-540-27099-X_14 . hal-01633392

\section{HAL Id: hal-01633392 \\ https://hal.science/hal-01633392}

Submitted on 12 Nov 2017

HAL is a multi-disciplinary open access archive for the deposit and dissemination of scientific research documents, whether they are published or not. The documents may come from teaching and research institutions in France or abroad, or from public or private research centers.
L'archive ouverte pluridisciplinaire HAL, est destinée au dépôt et à la diffusion de documents scientifiques de niveau recherche, publiés ou non, émanant des établissements d'enseignement et de recherche français ou étrangers, des laboratoires publics ou privés. 


\title{
Treating Moving Interfaces in Thermal Models with the C-NEM
}

\author{
Julien Yvonnet*, David Ryckelynck, Philippe Lorong, and Francisco \\ Chinesta \\ Laboratoire de Mécanique des Systèmes et des Procédés, UMR 8106 CNRS \\ ENSAM-ESEM, 151 boulevard de l'Hôpital, F-75013 Paris, France.
}

\begin{abstract}
This paper deals with the description of a new numerical simulation technique based in the constrained natural element method, a novel meshless method, able to compute multiphase thermal problems with moving interfaces without requiring the frequent mesh updating characteristics of interfaces tracking finite element techniques. This strategy combines some of the ideas of the natural element method with a particular treatment of the moving boundaries and interfaces involving discontinuities of some fields.
\end{abstract}

\section{Introduction}

Phase boundaries represent material interfaces across several fields which may exhibit sharp gradients, and even discontinuities. A wide range of numerical methods have been developed for treating these problems according to the pertinent physics and assumptions about the interface [LER00]. When a sharp interface is considered, its motion is governed by the jump in the temperature gradient normal to the phase boundary and is accompanied by the latent heat effects (Stefan condition). In order to satisfy these conditions the most common approach lies in tracking explicitly the interface motion. Within the interface tracking approach two main alternatives exist: the moving mesh methods and the mixed Eulerian-Lagrangian methods. Moving finite element mesh methods conform element boundaries to the interfaces as it evolves. Although these methods are very accurate, they are limited by the severe mesh distortion. Thus, frequent remeshing is needed, with the associated field projections between successive meshes. Moreover, remeshing is, even today, a delicate task in the $3 \mathrm{D}$ case. To alleviate remeshing efforts a number of Eulerian-Lagrangian methods have been developed recently that track the interface while solving the equations on a fixed grid [UMS99]. Many of these methods effectively smear the discontinuity over a few grid cells, and are therefore not capable of representing the true discontinuity across the interface.

\footnotetext{
* julien.yvonnet@paris.ensam.fr
} 
A new approach for representing localized behaviours has recently emerged in the field of the finite element method, known as the partition of unity method [MBA96]. The main idea is to extend the classical approximation considering the product of the standard shape functions and local enrichment functions. The extended finite element method (X-FEM) is a variation on this framework. Recently, the X-FEM has been coupled to the Level Set Method [SSO94] to represent interface topologies [SCM01]. In this way, the discontinuity evolution can be properly represented on a fixed background mesh, just by adding an appropriate enrichment in the functional approximation in the elements concerned by the moving discontinuity [JCD02]. However, when the material in which the interface is moving, is subjected to large displacements, an updated Lagrangian description could be a better choice. When the background mesh evolves, remeshing will be required also to avoid too high background mesh distortions.

To alleviate dependance to the mesh and to provide smoother shape functions, the use of the meshfree or meshless methods is investigated. The meshless methods discretize a continuum body by a finite number of particles (or nodes) and the field of interest is interpolated under these nodes without the aid of an explicit mesh. Many meshless methods have been proposed, including the smooth particle hydrodynamics (SPH) [LUC77], the diffuse element method (DEM) [NTV92], the element free Galerkin (EFG) [BLG94], the reproducing kernel particle method (RKPM) [LJZ95], the HP clouds DOD96 and the partition of unity method (PUM) [MBA96].

The introduction of moving discontinuities in these meshless methods can present difficulties for the following reasons: (i) The quality of the approximation as well as the conditioning of the global system is pathologically dependant from the size of the support of the shape functions; (ii) Imposition of essential boundary conditions needs particular treatments; (iii) Integration is not accurately defined, and (iv) The physical discontinuity across the interfaces must be introduced accurately. In order to overcome these different problems, we propose the use of the constrained natural element method (CNEM) [YRa03] [YRb03] for treating thermal models involving moving interfaces. This approach is an extension of the natural element method [SMB98] in which both trial and test functions are constructed on the basis of the Voronoi based interpolants [SIB80] [HSU02], verifying the Kronecker delta property and whose support is defined by the union of the Delaunay spheres passing through the visible nodes. In the C-NEM, the introduction of a visibility criterion and its related constrained Voronoi diagram preserves the appealing properties of the NEM in any geometry (convex or not) and allows easily the introduction of material discontinuities. In a former paper the C-NEM has been sucessfully applied in some problems involving non convex domains, discontinuities and cracks [YRb03].

The structure of this work is as follow: in section 2, we introduce a simple mathematical model of a thermal problem involving a moving interface. Actually, it turns out that it results the standard Stefan problem [VUI93]. In 
section 3, the C-NEM is summarized, and it will be applied in section 4 to discretize the weak formulation of the non-linear Stefan problem. A numerical benchmark is presented in section 5, which allows us to conclude about the accuracy of the proposed technique by comparing the numerical results with the exact solution of the problem.

\section{Problem Formulation}

Let $\Omega \subset \Re^{2}$ be a bounded domain and $T$ the temperature field. On the domain boundary $\Gamma \equiv \partial \Omega$ the temperature or the thermal flux are prescribed. We will denote by $\Gamma_{1}$ the domain boundary where the temperature is known $T\left(\mathbf{x} \in \Gamma_{1}, t\right)=\bar{T}(\mathbf{x}, t)$ and by $\Gamma_{2}$ the domain boundary where the heat flux $\bar{q}$ is imposed. The thermal model is defined in the time interval $\left[0, t_{\max }\right]$. The initial temperature $T(\mathbf{x}, t=0)=T_{0}$, where $T_{0}$ is assumed to be higher than the material melting temperature $T_{m}$. At time, $t=0$, a part of the domain boundary $\Gamma_{1}$ is suddenly submitted to a temperature $T_{1}<T_{m}$. A moving solidification front $\Gamma_{I}$ is then generated, whose position evolves in time, i.e. $\Gamma_{I}(t)$, dividing the domain $\Omega$ in two regions $\Omega_{1}(t)$ (containing the solid phase at time $t$ ) and $\Omega_{2}(t)$ (which contains the liquid phase) as shown in Figure 2.1. For a sake of simplicity we will consider, from now on, a homogeneous and isotropic thermal model in both phases.

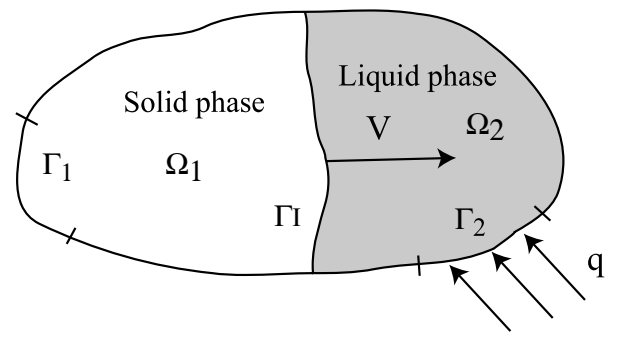

Figure 2.1. Two phases problem.

The heat transfer model is defined in each phase, neglecting volumetric source terms, by:

$$
\begin{cases}c_{1} \frac{\partial T(\mathbf{x}, t)}{\partial t}=\nabla \cdot\left(k_{1} \nabla T\right) & \text { in } \Omega_{1}(t) \\ c_{2} \frac{\partial T(\mathbf{x}, t)}{\partial t}=\nabla \cdot\left(k_{2} \nabla T\right) & \text { in } \Omega_{2}(t)\end{cases}
$$

where $c_{1}$ and $c_{2}$ are the volumetric heat capacities of both phases, being $k_{1}$ and $k_{2}$ their thermal conductivities. The associated initial and boundary conditions are: 


$$
\begin{cases}T(\mathbf{x}, t=0)=T_{0} & \forall \mathbf{x} \in \Omega \\ T(\mathbf{x}, t)=\bar{T}(\mathbf{x}, t) & \forall \mathbf{x} \in \Gamma_{1}, \forall t \in\left[0, t_{\text {max }}\right] \\ -k \nabla T(\mathbf{x}, t) \cdot \mathbf{n}=\bar{q}(\mathbf{x}, t) & \forall \mathbf{x} \in \Gamma_{2}, \forall t \in\left[0, t_{\text {max }}\right]\end{cases}
$$

The evolution of the interface $\Gamma_{I}(t)$ is described by a Stefan condition:

$$
\mathbf{V}\left(\mathbf{x} \in \Gamma_{I}(t)\right)=\frac{|[q]|}{L} \mathbf{n}_{I}(\mathbf{x})
$$

where $\mathbf{V}$ is the interface velocity, $L$ is the volumetric latent heat of fusion, $\mathbf{n}_{I}(\mathbf{x})$ is the normal vector to the interface at point $\mathbf{x}$ which is assumed to point into the liquid phase, and $[[q]]$ the thermal flux jump across the interface $\Gamma_{I}(t)$, i.e.

$$
|[q]|=\left(\left.k_{1} \nabla T\right|_{\Gamma_{I}^{-}(t)}-\left.k_{2} \nabla T\right|_{\Gamma_{I}^{+}(t)}\right) \mathbf{n}_{I}
$$

The additional constraint prescribed on the interface $\Gamma_{I}(t)$ is:

$$
T(\mathbf{x}, t)=T_{m} ; \quad \forall \mathbf{x} \in \Gamma_{I}(t)
$$

where $T_{m}$ is the melting temperature.

\section{The Constrained Natural Element Method (C-NEM)}

In this section, the utility of the C-NEM to describe moving interfaces and discontinuities in a fixed cloud of nodes is discussed. After a brief review of the Voronoi-based interpolants, we introduce the constrained Voronoi diagram which is used for computing the shape functions in any domain.

\subsection{Natural Neighbor Interpolation}

We briefly touch upon the foundation of Sibson's natural neighbor coordinates (shape functions) that are used in the natural element method. For a more in-depth discussion on the Sibson interpolant and its application for solving second-order partial differential equations, the interested reader can refer to Braun and Sambridge [SBM95], and Sukmar et al. [SMB98]. The NEM interpolant is constructed on the underlying Voronoi diagram. The Delaunay tesselation is the topological dual of the Voronoi diagram.

Consider a set of nodes $S=\left\{n_{1}, n_{2}, \ldots, n_{N}\right\}$ in $\Re^{2}$. The Voronoi diagram is the subdivision of $\Re^{2}$ into regions $T_{i}$ (Voronoi cells) defined by:

$$
T_{i}=\left\{\mathbf{x} \in \Re^{2}: d\left(\mathbf{x}, \mathbf{x}_{i}\right)<d\left(\mathbf{x}, \mathbf{x}_{j}\right), \forall j \neq i\right\}, \quad \forall i
$$

The Sibson coordinates of $\mathbf{x}$ with respect to a natural neighbor $n_{i}$ (see Figure 3.2) is defined as the ratio of the overlap area (volume in 3D) of their Voronoi cells to the total area (volume in $3 \mathrm{D}$ ) of the Voronoi cell related to point $\mathbf{x}$ : 

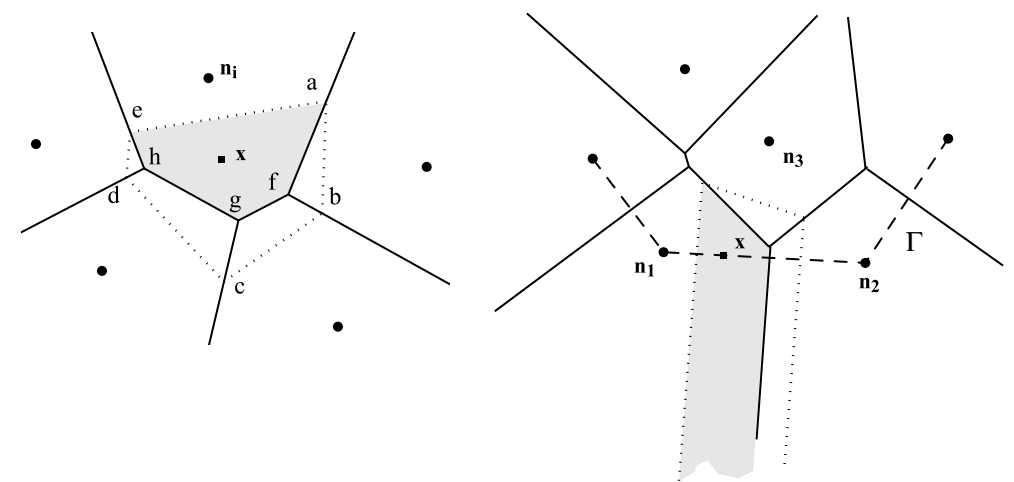

Figure 3.2. Construction of the Sibson shape functions.

$$
\phi_{i}(\mathbf{x})=\frac{\text { Area }(\text { afghe })}{\text { Area }(\text { abcde })}
$$

If the point $\mathbf{x}$ coincides with the node $n_{i}$, i.e. $\left(\mathbf{x}=\mathbf{x}_{i}\right), \phi_{i}\left(\mathbf{x}_{i}\right)=1$, and all other shape functions are zero, i.e. $\phi_{j}\left(\mathbf{x}_{i}\right)=\delta_{i j}\left(\delta_{i j}\right.$ being the Kroenecker's delta). The properties of positivity, interpolation, and partition of unity are then verified [SMB98]:

$$
\left\{\begin{array}{l}
0 \leq \phi_{i}(\mathbf{x}) \leq 1 \\
\phi_{i}\left(\mathbf{x}_{j}\right)=\delta_{i j} \\
\sum_{i=1}^{n} \phi_{i}(\mathbf{x})=1
\end{array}\right.
$$

The natural neighbor shape functions also satisfy the local coordinate property [SIB80], namely:

$$
\mathbf{x}=\sum_{i=1}^{n} \phi_{i}(\mathbf{x}) \mathbf{x}_{i}
$$

which combined with Eq. (3.3), implies that the natural neighbor interpolant spans the space of linear polynomials (linear completeness).

Natural neighbor shape functions are $C^{\infty}$ at any point except at the nodes, where they are only $C^{0}$, and on the boundary of the Delaunay circles (spheres in $3 \mathrm{D}$ ) where they are only $C^{1}$, because of the discontinuity in the neighbors nodes across these boundaries.

Another important property of this interpolant is the ability to reproduce linear functions over the boundary of convex domains. The proof can be found in Sukumar et al. [SMB98]. An illustration is depicted in Figure 3.2 (b): as the areas associated to points on the boundary become infinite, the contribution of internal points vanish in the limit when the point approaches the convex boundary, and the shape functions associated with nodes $n_{1}$ and $n_{2}$ become linear on the segment $\left(n_{1}-n_{2}\right)$. This is not true in the case of non convex 
boundaries, and the next section focuses in an approach to circumvert this difficulty.

Consider an interpolation scheme for a scalar function $T(\mathbf{x}): \Omega \subset \Re^{2} \rightarrow$ $\Re^{2}$, in the form:

$$
T^{h}(\mathbf{x})=\sum_{i=1}^{n} \phi_{i}(\mathbf{x}) T_{i}
$$

where $T_{i}$ are the nodal temperatures at the $n$ natural neighbor nodes, and $\phi_{i}(\mathbf{x})$ are the shape functions associated with each neighbor node. It is noted that Eq. (3.5) defines a local interpolation scheme. Thus, the trial and test functions used in the discretization of the variational formulation describing the thermal problem treated in this paper take the form of Eq. (3.5).

\subsection{The Constrained Natural Element Method}

Constrained Voronoi Diagram. It was proved in [YRb03] [SMB98] and [CCC02] that spurious influences between "non-visible" nodes and lost of linearity in the interpolation along boundaries of non convex domains appear in the framework of the NEM. In order to avoid this drawback and to recover all properties of the method for any geometry (including non convex domains containing cracks or involving field discontinuities), a visibility criterion is introduced in order to restrict influent nodes among natural neighbors. The computation of the shape functions is done on the basis of the so-called constrained (or extended) Voronoi diagram (CVD), introduced by Seidel in [SEI88]. The constrained Voronoi cells are defined formally by:

$$
\begin{gathered}
T_{i}^{C}=\left\{\mathbf{x} \in \Re^{n}: d\left(\mathbf{x}, \mathbf{x}_{i}\right)<d\left(\mathbf{x}, \mathbf{x}_{j}\right),\right. \\
\left.\forall j \neq i, S_{x \rightarrow n_{i}} \cap \Gamma=\emptyset, S_{x \rightarrow n_{j}} \cap \Gamma=\emptyset\right\}
\end{gathered}
$$

where $\Gamma$ is the domain boundary, composed with segments $l_{i} \in L, L$ being a set of segment in the plane, and $S_{a \rightarrow b}$ denotes the segment between the points $a$ and $b$.

A generalization of the constrained Delaunay triangulation to 3D doesn't exist without adding new nodes, as shown in [SCH28]. Nevertheless, some techniques for constructing 3D constrained Delaunay tesselations are available and provided in [SMB98],[SHE00] by addition of Steiner points.

The Constrained Natural Element Approximation. In order to solve partial differential equations defined in non convex domains, or to reproduce some functional discontinuities, we consider the following approximation of both the trial and the test functions:

$$
T^{h}(\mathbf{x})=\sum_{i=1}^{V} \phi_{i}^{C}(\mathbf{x}) T_{i}
$$


where $V$ is the number of natural neighors visible from point $\mathbf{x}$ and $\phi_{i}^{C}$ is the constrained natural neighbor shape function related to the $\mathrm{i}$-th node at point $\mathrm{x}$. The computation of the C-n-n (constrained natural neighbor) shape functions is similar to the natural neighbor shape function, when one proceed using the constrained Voronoi diagram introduced previously. It was shown in [YRa03] and [YRb03] that the use of the constrained Voronoi diagram does not affect the properties of the NEM interpolation, allowing the extension of the linearity of the shape functions on the convex domains boundaries, to any geometry, convex or not.

The ability of the C-NEM for treating problems involving cracks has been illustrated in [YRb03]. In the present paper, we focus on its application in the context of a moving interface defining two domains with different thermal properties. Thus, defining at time $t$ two CVD (constrained Voronoi diagrams) of $\Omega_{1}(t)$ and $\Omega_{2}(t)$, both with respect to the interface $\Gamma_{I}(t)$, it can be proved that the interpolated temperature field is $C^{1}$ everywhere, except at the nodes and on the interface $\Gamma_{I}(t)$ where it is only $C^{0}$. Thus, this interpolation seems to be appropriate to simulate the Stefan problem considered in this paper.

To illustrate this behavior, we consider the situation depicted in Figure 3.3 , where the point $\mathbf{x}$ moves from $\Omega_{1}$ to $\Omega_{2}$. If $\mathbf{x}$ is in $\Omega_{1}$, the interpolated field is constructed from Eq. (3.7) using the neighbor visible nodes from point $\mathbf{x}\left(\Gamma_{I}\right.$ is assumed opaque). If $\mathbf{x}$ is on $\Gamma_{I}$, according to the previous discussion, the interpolated field is strictly linear because it only depends on the two neighbor nodes located on $\Gamma_{I}$. Finally, when $\mathbf{x}$ is in $\Omega_{2}$, the interpolated field is defined using the visible neighbor and visible nodes from point $\mathbf{x}\left(\Gamma_{I}\right.$ being opaque). The continuity of the interpolated field is then guaranteed, but a discontinuity appears in the field derivatives, because of a sudden change in the neighbor nodes across the interface. We can then reproduce the temperature field continuity, as well as the expected flux discontinuity on the interface.

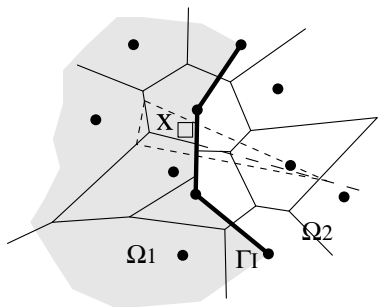

(a)

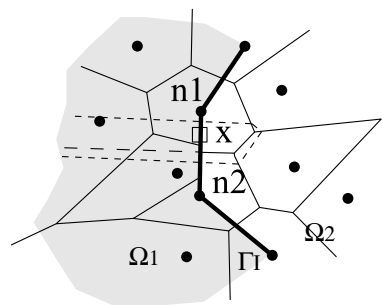

(b)

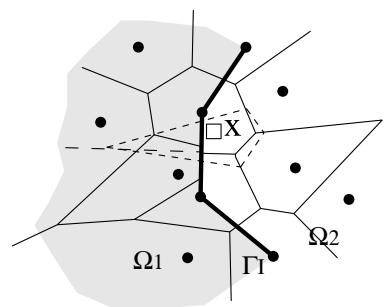

(c)

Figure 3.3. Reproducing discontinuous derivatives using the constrained Voronoi diagram. 


\section{C-NEM Discretization}

Assuming that in our example $\Gamma_{2} \equiv \Gamma_{I}$, the weak formulation associated with Eq. (2.1) results:

Find $T \in H^{1}(\Omega)$ verifying $T=\bar{T}$ on $\Gamma_{1}$ such that:

$$
\int_{\Omega} c \frac{\partial T}{\partial t} \delta T d \Omega=-\int_{\Omega} k \nabla T \cdot \nabla \delta T d \Omega+\int_{\Gamma_{I}(t)}|[q]| \delta T d \Gamma, \quad \forall \delta T \in H_{0}^{1}(\Omega)
$$

Where $H^{1}(\Omega)$ and $H_{0}^{1}(\Omega)$ are the usual Sobolev functional spaces. Substituting the trial and test functions (both approximated in the C-NEM framework) in the above equation and using the arbitrariness of the field $\delta T$, the following system of equations is obtained:

$$
\mathbf{C} \dot{\mathbf{T}}+\mathbf{K T}=\mathbf{F}
$$

where $\mathbf{T}$ is the vector containg the unknown nodal temperatures. For time discretization, we consider the solution on the time interval $\left[0, t_{\max }\right]$, partitioned into steps as $\left[t^{n}, t^{n+1}\right]$ and the generalized trapezoidal time stepping algorithm characterized by the parameter $\alpha$ :

$$
\frac{\partial T^{n+1}}{\partial t}=\frac{T^{n+1}-T^{n}-(1-\alpha) \Delta t \frac{\partial T^{n}}{\partial t}}{\alpha \Delta t}
$$

where $\frac{\partial T^{0}}{\partial t}$ is initialized by setting:

$$
\int_{\Omega} c \frac{\partial T^{0}}{\partial t} \delta T d \Omega=-\int_{\Omega} k \nabla T^{0} \cdot \nabla \delta T d \Omega \quad \forall \delta T \in H_{0}^{1}(\Omega)
$$

which leads, after applying the discretization scheme described in Eq. (3.5), to:

$$
\mathbf{C} \frac{\partial \mathbf{T}^{0}}{\partial t}=-\mathbf{K} \mathbf{T}^{0}
$$

The stabilized conforming nodal integration proposed by Chen et al. in [CWY01] is employed for the numerical integration of $\mathbf{K}$ (see our former work [YRb03] for more details). This technique, based on the assumed strain method, has been used to reduce significantly the integration errors, and allows to satisfy the patch test exactly in the context of the natural neighbor interpolation, which is not the case if a standard Gauss quadrature scheme is used [CCC02]. In the context of the C-NEM, the representative domains related to each node used in such techniques to define the assumed gradient, are the constrained Voronoi cells depicted in figure 3.3, which are accurately defined everywhere, and especially in the interface neighborhood.

A lumped mass matrix $\tilde{\mathbf{C}}$ is computed making use of the constrained Voronoi cells areas as nodal weights. 
The iteration procedure is defined as:

Knowing $\mathbf{T}^{n}$ and $|[q]|^{n}$ at time $t^{n}$, the non-linear problem associated with Eq. (4.1) results in finding $\mathbf{T}^{n+1}$ and $|[q]|^{n+1}$ such that Eqs. (2.5) and (4.1) are satisfied. For this purpose we proceed as follows:

1. Compute the interface velocity $\mathbf{V}^{n}(\mathbf{x})$ using Eq. (2.3) and update the interface position at time $t^{n+1}$ using the forward Euler formula:

$$
\mathbf{x}_{J}^{n+1}=\mathbf{x}_{J}^{n}+\Delta t \mathbf{V}^{n}\left(x_{J}^{n}\right)
$$

where $\mathbf{x}_{J}$ are the nodes defining the interface.

2. Update locally the constrained Voronoi diagram and the shape functions associated with integration points in the interface neighborhood. Then, we compute $\tilde{\mathbf{C}}^{n+1}$ and $\mathbf{K}^{n+1}$.

3. Solve Eq. (4.1) using a Newton-Raphson procedure where the tangent matrix is computed numerically.

4. Repeat while $t^{n+1}<t_{\max }$.

An alternative scheme using the Latin method [LAD98] in the extended finite element framework can be found in Merle and Dolbow [MED02].

\section{Numerical Example}

In this section, we illustrate the potentiality of the proposed technique simulating a two-phases Stefan problem. The problem is essentially one-dimensional, but we solve it here in two dimensions to underline the outstanding features of the method.

The Stefan problem models the one-dimensional freezing of a semi-infinite domain $(x \geq 0)$. The initial temperature $T_{0}$ is assumed constant in the whole domain, being higher than the melting temperature $T_{m}$. At time $t=0$ the temperature at the left boundary $x=0$ is suddenly prescribed to a value $T_{1}$ lower than the melting point, originating a solidification front that progresses from the boundary $x=0$ in the $x$ direction. The exact flow front position $x_{f}(t)$ is given by:

$$
x_{f}(t)=2 \lambda \sqrt{\beta_{s} t}
$$

where $\beta_{s}=k_{s} / c_{s}$ is the thermal diffusivity of the solid phase, and the constant $\lambda$ satisfies the following relationship:

$$
\frac{e^{-\lambda^{2}}}{\operatorname{erf}(\lambda)}=\frac{k_{l} \sqrt{\eta}\left(T_{0}-T_{m}\right) e^{-\eta \lambda^{2}}}{k_{s}\left(T_{m}-T_{1}\right) \operatorname{erfc}(\lambda \sqrt{\eta})}+\frac{\lambda L \sqrt{\pi}}{c_{s}\left(T_{m}-T_{1}\right)}
$$

with $\eta=\beta_{s} / \beta_{l}$ being the ratio of the thermal diffusivities and where $k_{l}$ represents the liquid phase conductivity. The temperature field in the solid phase $0 \leq x \leq x_{f}(t)$ is then: 


$$
T(x, t)=T_{1}+\frac{T_{m}-T_{1}}{\operatorname{erf}(\lambda)} \operatorname{erf}\left(\frac{x}{2 \sqrt{\beta_{s} t}}\right)
$$

and in the liquid phase $x \geq x_{f}(t)$ :

$$
T(x, t)=T_{0}-\frac{T_{0}-T_{m}}{\operatorname{erfc}(\lambda \sqrt{\eta})} \operatorname{erfc}\left(\frac{x}{2 \sqrt{\beta_{l} t}}\right)
$$

In the present investigation, we use the water-satured sand thermal properties provided in [LYO81] and listed in Table I. $T_{1}$ and $T_{0}$ were set to -10 and $4.0{ }^{0} C$ respectively $(\lambda=0.3073)$. We simulate the evolution of the temperature field in $\Omega=[0,1] \times[0,0.5] \mathrm{cm}$. In order to use the inifinite domain solution as reference solution, the temperature at $x=1$ is prescibed to its expected value according to Eq. (5.4)

\begin{tabular}{|c|c|c|c|}
\hline Properties & Solid & Liquid & \\
\hline Volumetric heat capacity $\left(\mathrm{cal}^{0}{ }^{0} \mathrm{C}^{-1} \mathrm{~cm}^{-3}\right)$ & 0.49 & 0.62 & \\
\hline Thermal conductivity $\left(\right.$ cal. $\left.\mathrm{cm}^{-1} \mathrm{~s}^{-1} \cdot{ }^{0} \mathrm{C}^{-1}\right)$ & $9.6 .10^{-3}$ & $6.9 .10^{-3}$ & \\
\hline Melting temperature $\left({ }^{0} C\right)$ & & & 0.0 \\
\hline Volumetric latent heat of fusion $\left(\mathrm{cal} . \mathrm{cm}^{-3}\right)$ & & & 19.2 \\
\hline
\end{tabular}

Table 5.1. Thermal properties of the water saturated sand.

In a first test, we consider in the domain $\Omega$ a $20 \times 10$ uniform grid and $\Delta t$ $=2 \mathrm{~s}$. Figure 5.4 compares the computed interface position and the exact one. An excellent accuracy can be noticed, as depicted in figure 5.5, where the error in the front position is represented. Figure 5.6 shows the temperature profile at different times. We can point out that the discontinuity in the temperature gradient is accurately obtained, being in excellent agreement with the exact solution. The external boundary conditions are given by: $T(t)=T_{1}$ on $(x=0)$, $T(t)=T_{e x}$ given by Eq. (5.4) on $(x=1)$ and $-k \nabla T \cdot \mathbf{n}=0$ on $(\mathrm{y}=0)$ and $(\mathrm{y}=0.5)$. The initial condition is given by $T(t=0)=T_{0}$ in $\Omega$.

In a second test, we consider the domain $\Omega$ containing 200 nodes distributed at random. The purpose of the present test is to investigate the meshless feature of the technique, in which due to its meshless character no geometrical restrictions concerning the relative nodal positions are involved. Thus, neither the background nodal distribution nor the relatinve position of the nodes defining the moving interface with respect to the background nodes, induce a lack of accuracy when high distortions in the Delaunay mesh, used to compute the Voronoi diagram, takes place. This is the main difference between the proposed strategy and the standard finite element method whose accuracy depends significantly on the geometrical quality of the mesh. Moreover, this test approaches the situations encountered when the material is also moving, inducing highly irregular nodal densities and high background mesh 


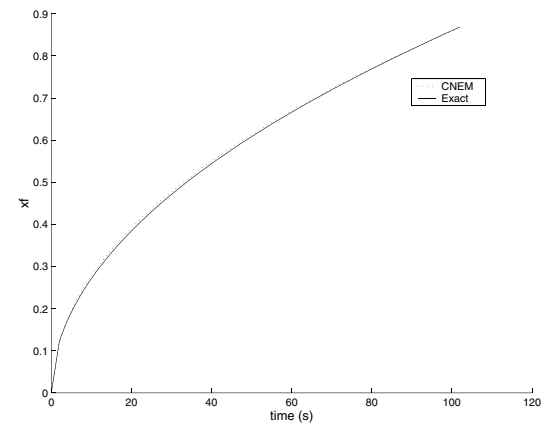

Figure 5.4. Computed C-NEM front position versus the exact solution using a $20 \times 10$ regular grid.

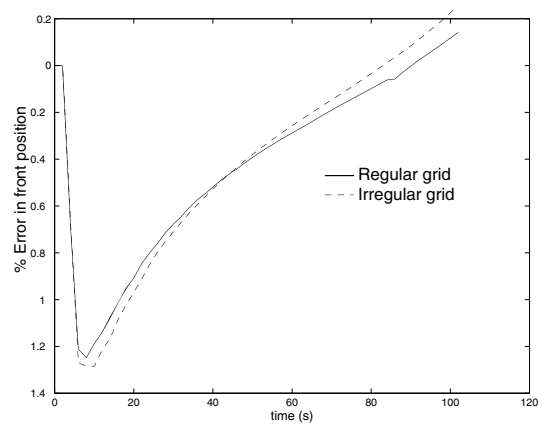

Figure 5.5. Error in the front position.

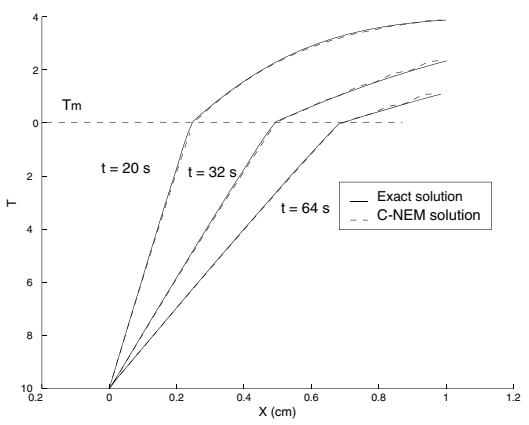

Figure 5.6. Temperature profiles along the line $\mathrm{y}=0.25$ using a regular grid.

distortions. Figure 5.7 depicts the cloud of nodes and the interface position as well as the associated constrained Voronoi cells. Remarkably, despite of the very irregular nodal distribution and density, we can notice that the interface is not distorted as it moves through the domain. From Figs. 5.5 and 5.8 we can conclude that the accuracy is not affected significantly by the resgularity on the nodal distribution. In Figure 5.9 some temperature profiles along the line $y=0.25$ are depicted, from which an excellent accuracy can be noticed.

\section{Conclusion}

In this paper, the salient features of the C-NEM meshless method are used for treating thermal problems involving moving interfaces. The use of the constrained Voronoi diagram allows to introduce the desired discontinuities in the gradient of the solution without any enrichment. In the C-NEM framework, essential boundary conditions can be imposed directly, due to the strict linearity of the shape functions over the boundaries (convex or not) and the 

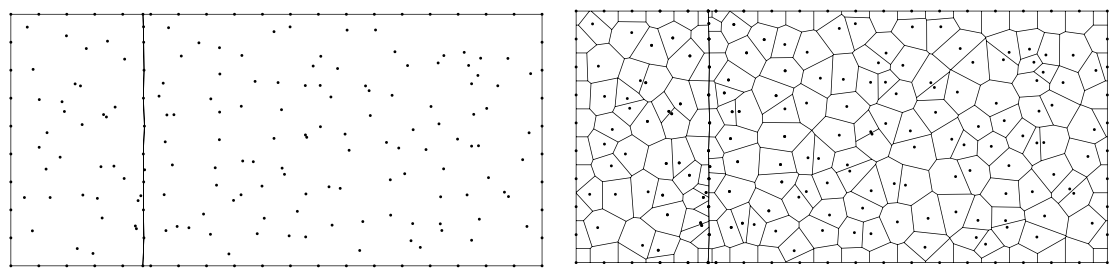

$\mathrm{t}=8 \mathrm{~s}$
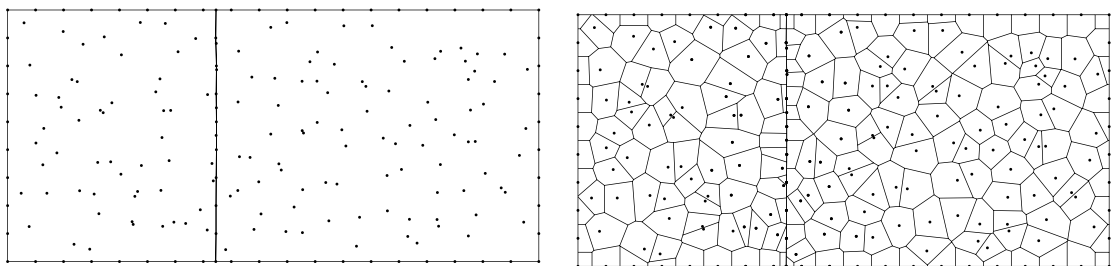

$\mathrm{t}=20 \mathrm{~s}$
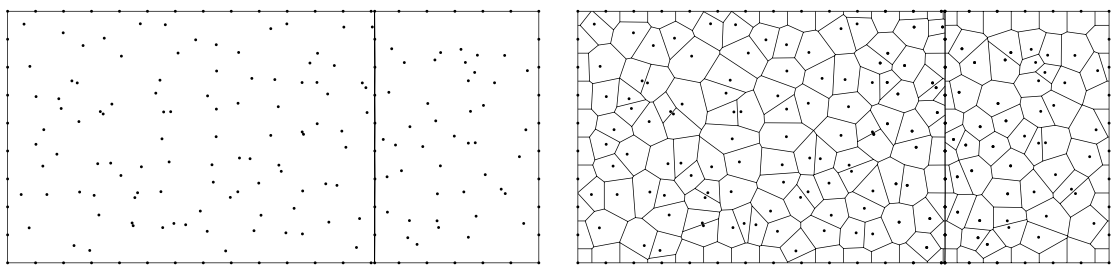

$\mathrm{t}=64 \mathrm{~s}$
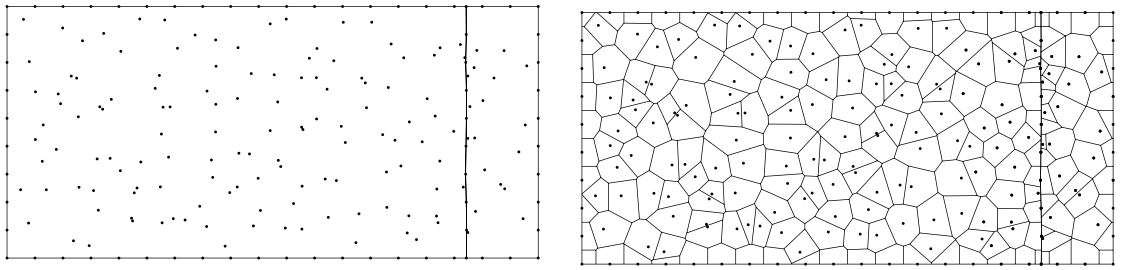

$t=100 s$

Figure 5.7. Computed interface position using an irregular cloud of nodes: (a) Cloud of nodes and interface position; (b) Constrained Voronoi cells.

respect of the Kronecker delta property. No user-defined parameter is involved in the shape functions support size, the support being defined like the union of the Delaunay spheres passing through a node and its neighbor visible nodes. A stabilized conforming nodal integration is computed over the constrained Voronoi cells to enhance accuracy. The most outstanding quality of this technique is the ability for introducing discontinuities located on a line (surface in 3D) defined by another set of nodes that move through a fixed (or also moving) cloud of nodes which constitutes a background scattered of nodes. 


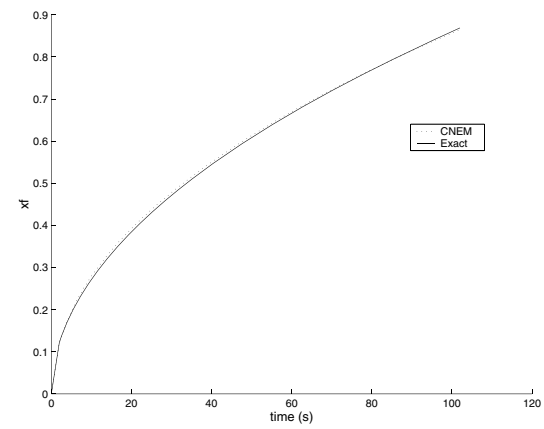

Figure 5.8. C-NEM interface position versus the exact solution using an irregular grid.

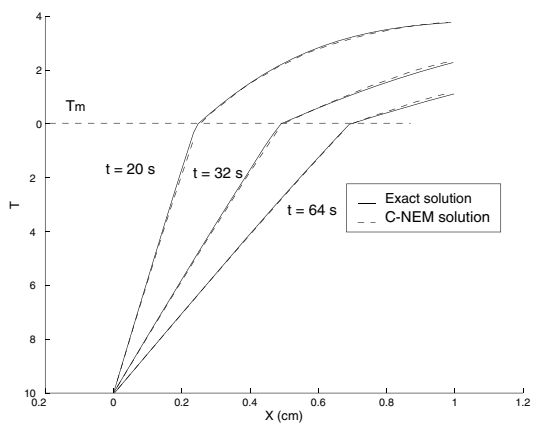

Figure 5.9. Temperature profiles along the line $y=0.25$ using an irregular grid.

The meshless character of this technique allows to proceed without remeshing (in a finite element sense), even for highly irregular nodal distributions. The method is relatively simple and delivers similar level of accuracy than r-adaptative or the partition of unity finite element schemes. The technique seems promising for the simulation of arbitrary and moving discontinuities over an unstructred fixed or also moving set of nodes.

\section{References}

[BLG94] Belytschko T., Lu Y.Y., Gu L.: Element-free Galerkin methods. International Journal for Numerical Methods in Engineering 37, 229-256 (1994)

[CWY01] Chen J.S., Wu C.T., Yoon Y.: A stabilized conforming nodal integration for Galerkin mesh-free methods. International Journal for Numerical Methods in Engineering, 50, :435-466 (2001)

[CCC02] Cueto E., Cegoñino J., Calvo B., Doblaré M.: On the imposition of essential boundary conditions in Natural neighbor Galerkin Methods. Communications in Numerical Methods in Engineering, 19, 361-376 (2002)

[DOD96] Duarte C.A., Oden J.T.: An H-p adaptative method using clouds. Computer Methods in Applied Mechanics and Engineering 139, 237-262 (1996)

[HSU02] Hiyoshi H., Sugura K.: Improving continuity of Voronoi-based interpolation over Delaunay spheres. Computational Geometry, 22, 167-183 (2002)

[JCD02] Ji H., Chopp D., Dolbow J.E.: A hybrid finite element/level set method for modeling phase transformations. International journal for numerical methods 54, 1209-1233 (2002)

[LAD98] Ladevèze P.: Non Linear Computational Structural Mechanics. Springer:, New York (1998)

[LER00] Lewis, R., Ravindran, K.: Finite element simulation of metal casting. International journal for numerical methods in engineering, 47, 93-102 (2000) 
[LJZ95] Liu W.K., Jun S., Zhang Y.F.: Reproducing Kernel Particle Methods. Int. J. Numer. Methods Fluids 21, 1081-1106 (1995)

[LUC77] Lucy L.B.: A numerical approach to the testing of fusion process. The astronomic journal 88, 1013-1024 (1977)

[LYO81] Lynch D., O'Neill K.: Continuously deforming finite elements for the solution of parabolic problems, with and without phase change. International Journal for Numerical Methods in Engineering, 17, 81-96 (1981)

[MBA96] Melenk, J.M., babuška, I.: The partition of unity finite element method: basic theory and applications. Computer Methods in Applied Mechanics and Engineering, 139, 289-314 (1996)

[MED02] Merle R., Dolbow J.E.: Natural neighbor Galerkin methods. Solving thermal and phase change with the extended finite element method, 28(5), 339-350 (2002)

[NTV92] Nayroles B., Touzot G., Villon P.: Generalizing the finite element method: diffuse approximation and diffuse elements. Computational mechanics 10, 307-318 (1992)

[UMS99] Udaykumar, H., Mittal, R, Shyy W.: Computation of solid-liquid phase fronts in the sharp interface limit on fixed grids. Journal of computational physics, 153, 535-574 (1999)

[SBM95] Sambridge M., Braun J., McQueen M.: Geophisical parameterization and interpolation of irregular data using natural neighbors. Geophys. i. J. Int, 122 ,

[SCH28] Schönhardt E.: Uber die zerlegung von dreieckspolyedern in tetraeder. Math. Annalen, 98, (1928) 837-857 (1995)

[SEI88] Seidel R.: Constrained Delaunay triangulations and Voronoi diagrams with obstacles In: "1978-1988 Ten Years IIG" 178-191. (1988)

[SHE98] Shewchuck J.R.: Tetrahedral mesh generation by delaunay refinement. In: Proceedings of the fourteenth annual symposium on computational geometry, Minneapolis, Minnesota, june 1998; pp. 86-95, association for computing machinery (1988)

[SHE00] Shewchuck J.R.: Sweep algorithms for constructing higher-dimensional constrained Delaunay triangulations. In: Proceedings of the sixteenth annual symposium on computational geometry, Hong-Kong, june 2000; pp. 350-359, association for computing machinery (2000)

[SIB80] Sibson R.: A vector Identity for the Dirichlet tesselations. Math. Proc. Camb. Phil. Soc., 87, 151-155 (1980)

[SCM01] Sukumar N., Chop D., Moës N., Belytschko T.: Modeling holes and inclusions by level sets in the extended finte element method. Computer methods in appled mechanics and engineering 190, 6183-6200 (2001)

[SMB98] Sukumar N., Moran B., Belytschko T.: The natural elements method in solid mechanics. International Journal for Numerical Methods in Engineering, 43, 839-887 (1998)

[SMSB01] Sukumar N., Moran B., Semenov Y., Belikov V.V.: Natural neighbor Galerkin methods. International Journal for Numerical Methods in Engineering, 50, 207-219 (2001)

[SSO94] Sussman M., Smereka P., Osher S.: A level set approach for computing solutions to incompressible two-phase flows. Journal of computational physics 114, 146-159 (1994)

[VUI93] Vuik C.:Some historical notes about the Stefan problem. In: CWI-tract 90 (CWI, Amsterdam) (1993) 
[YRa03] Yvonnet J., Ryckelynck D., Lorong P., Chinesta P.: Interpolation naturelle sur les domaines non convexes par l'utilisation du diagramme de Voronoi contraint - MÖthode des ÖlÖments C-Naturels. Revue EuropÖenne des éléments Finis 12(4), 487-509 (2003)

[YRb03] Yvonnet J., Ryckelynck D., Lorong P., Chinesta P.: A new extension of the natural element method for non convex and dscontinuous problems, the constrained natural element method (C-NEM). International Journal for Numerical Methods in Engineering, accepted. 